\title{
Aspectos produtivos e econômicos de poedeiras comerciais submetidas a diferentes densidades de alojamento
}

\author{
Pasquoal Carrazzoni de Menezes ${ }^{1}$, Verônica Fernandes Tenório Cavalcanti ${ }^{2}$, Evilda \\ Rodrigues de Lima ${ }^{3}$, Joaquim Evêncio Neto ${ }^{4}$
}

\footnotetext{
${ }^{1}$ Doutorando, PPGCV, Universidade Federal Rural de Pernambuco, Recife, PE, Brasil.

2 Médica Veterinária Autônoma, Recife, $P E$

${ }^{3}$ Departamento de Medicina Veterinária, Universidade Federal Rural de Pernambuco, Recife, PE, Brasil.

${ }^{4}$ Departamento de Morfologia e Fisiologia Animal, Universidade Federal Rural de Pernambuco. Av. Manoel de Medeiros, s/n. Dois Irmãos, CEP: 52.171-900, Recife, PE, Brasil.
}

RESUMO - Esta pesquisa foi realizada com o objetivo de avaliar os aspectos produtivos e econômicos de poedeiras comerciais (Gallus gallus) submetidas a diferentes densidades de alojamento. Foram alojadas 264 poedeiras em gaiolas com dimensões de $100 \mathrm{~cm} \times 50 \mathrm{~cm} \times 40 \mathrm{~cm}$ em delineamento inteiramente casualizado, com quatro grupos distribuídos em esquema fatorial $4 \times 4$, com 8, 10, 12 e 14 aves por gaiola, correspondendo a 625, 500, 416,6 e 357,14 $\mathrm{cm}^{2} /$ ave. As características avaliadas foram: produção de ovos, peso médio dos ovos, consumo de ração, massa dos ovos, conversão alimentar por dúzia e ovos imprestáveis para consumo humano. A densidade na gaiola teve efeito apenas sobre os ovos tipos extra e segunda e sobre a produção diária dos ovos tipo segunda, mas não influenciou as demais variáveis estudadas. Para poedeiras comerciais da linhagem Dekalb White, a utilização de gaiolas com a densidade de alojamento de 625, 500, 416,6 e 357,14 $\mathrm{cm}^{2} /$ ave na fase de produção não prejudica os parâmetros de qualidade da produção, sob os aspectos zootécnico e econômico.

Palavras-chave: densidade de alojamento, desempenho, gaiola, poedeiras

\section{Productive and economical aspects of laying hens submitted to different housing densities}

\begin{abstract}
The objective of this research was to study productive and economical aspects of laying hens (Gallus gallus) submitted to different housing densities. Two hundred and sixty-four laying hens were housed in cages with dimensions of $100 \mathrm{~cm} \times 50 \mathrm{~cm} \times 40 \mathrm{~cm}$ in a randomized complete design with four groups distributed in a $4 \times 4$ factorial arrangement, with 8, 10, 12 and 14 animals/cage, corresponding to 625, 500, 416,6 and 357,14 cm$/$ animal. The following characteristics were assessed: egg production, medium egg weight, feed intake, egg mass, feed conversion per dozen and inappropriate eggs for human consumption. Cage density only had an effect on the extra large and medium type eggs and on the daily production of medium type eggs, but did not influence the other variables studied. For laying hens of the Dekalb White lineage, the use of cages with housing densities of $625,500,416,6$ and 357,14 $\mathrm{cm}^{2} /$ animal in the production phase did not harm the quality parameters under the productive and economical aspects.
\end{abstract}

Key Words: cage, housing density, laying hens, performance

\section{Introdução}

A avicultura desenvolveu muito nos últimos anos, buscando novos sistemas de criação, visando maior produtividade em menor tempo, passando por processo de evolução técnica na genética, alimentação, manejo e sanidade, fatores considerados sustentáculos da avicultura como atividade econômica e importantes na produção de alimentos para a população (Albuquerque, 2004; Furlan et al., 2006).
Estudos realizados sobre a relação entre densidade ave/alojada das gaiolas utilizadas em poedeiras no período de produção e seus efeitos na produtividade, não encontraram diferenças sobre a produção de ovos/ave/dia por ave alojada (Wells, 1971; Craig \& Milliken, 1989; Lee, 1989; Carey et al., 1995). Resultados contrários foram relatados por Adams \& Craig (1985), Davami et al. (1987) e Okpokho et al. (1987) Garcia et al. (1993) quando observaram que o aumento da densidade ave/alojada na gaiola e a redução da área de comedouro ocasionou declínio na produção de ovos. 
As linhagens de poedeiras modernas diferenciam-se das antigas quanto ao temperamento, potencial produtivo, consumo de ração, ganho de peso, viabilidade e tipo dos ovos. Também pelo fato de que as aves, a cada ano, tornam-se mais precoces, com adiantamento da idade em que atingem a maturidade sexual, o que constitui um desafio para os técnicos avícolas estimularem o consumo de ração e o ganho de peso das frangas em cria/recria, principalmente em linhagens de baixo consumo de ração.

Considerando que a atividade de produção de ovos tem, nos últimos anos, apresentado grande evolução em todos os seus segmentos, tornando-se cada vez mais competitiva, é importante estar atento, pois é possível estar sempre empregando o máximo de todos os recursos disponíveis (Barbosa Filho et al., 2007). Neste sentido, pode-se esperar que, nos dias atuais, as aves apresentem melhores resultados de viabilidade em altas densidades de alojamento quando comparadas às pesquisas realizadas nas décadas anteriores. São necessários, portanto, estudos que permitam melhor compreensão das inter-relações entre os fatores técnicos e impacto econômico do estudo da densidade populacional em aves poedeiras e sua influência nos aspectos zootécnicos e econômicos. Assim, realizou-se este trabalho para avaliar os aspectos produtivos e econômicos de poedeiras comerciais submetidas a diferentes densidades.

\section{Material e Métodos}

Esta pesquisa foi desenvolvida nas instalações da Empresa Ingá Agropecuária Ltda., com sede na cidade de Belo Jardim, Pernambuco, distante $180 \mathrm{~km}$ a oeste da cidade de Recife, Pernambuco, Brasil, durante o período de fevereiro a julho de 2006. Foram utilizadas 264 poedeiras comerciais (Gallus gallus) com 24 semanas de idade, da linhagem Dekalb White, uniformizadas segundo as características física, de peso e aspectos sanitários e criadas em um único programa nutricional nas fases de cria e recria. Em seguida, as aves foram alojadas em gaiolas metálicas com duas subdivisões de $50 \mathrm{~cm} \times 50 \mathrm{~cm} \times 40 \mathrm{~cm}$, distribuídas em quatro grupos, a saber: 8 aves por gaiola ( $625 \mathrm{~cm}^{2} /$ ave); 10 aves por gaiola (500 $\mathrm{cm}^{2} /$ ave); 12 aves por gaiola (416,6 $\mathrm{cm}^{2} /$ ave) e 14 aves por gaiola (357,14 $\mathrm{cm}^{2} /$ ave).

As aves de todos os grupos foram submetidas ao mesmo programa nutricional e mesmo manejo seguindo as exigências da linhagem para cada semana de idade e as especificações fornecidas pelo produtor das pintinhas e constantes no manual de criação da Dekalb White. O aviário utilizado foi construído de material pré-moldado na sua estrutura e coberto com madeira e telhas de cimento amianto.
O piso é cimentado e as laterais abertas. Foram utilizadas 24 unidades experimentais equipadas com gaiolas metálicas, medindo $100 \mathrm{~cm} \times 50 \mathrm{~cm} \times 40 \mathrm{~cm}$ com duas subdivisões de $50 \mathrm{~cm} \times 50 \mathrm{~cm} \times 40 \mathrm{~cm}$ cada.

Foram utilizados bebedouros do tipo nipple, e comedouros tipo calha metálica, colocados frontalmente e externamente à gaiola. Durante a pesquisa, a temperatura ambiente no interior do galpão oscilou entre $19^{\circ} \mathrm{C}$ e $32^{\circ} \mathrm{C}$ e a umidade relativa do ar variou entre $85 \%$ e $90 \%$.

O fornecimento da ração ocorreu diariamente e o consumo variou entre $101 \mathrm{~g} /$ ave/dia (inicial) e $107 \mathrm{~g} /$ ave/dia (final) de acordo com a idade das aves. Foi utilizada ração para poedeiras comerciais com a seguinte composição: proteína total 17,69\%; gordura 4,11\%; fibra bruta 3,29\%; cinzas 13,07\%; cálcio 3,97\%; fósforo total 0,60\%; 2.790,00 kcal $/ \mathrm{kg}$.

A coleta dos ovos foi realizada diariamente, duas vezes por dia, durante 150 dias, anotando-se em formulários próprios as classificações de acordo com o número de ovos inteiros e quebrados, a presença ou ausência de trincamento de casca, os ovos perdidos (sem casca ou ovos com falhas severas que impediam sua comercialização). Os ovos caídos embaixo das gaiolas foram contados e recolhidos diariamente e considerados também nos resultados. Os ovos foram classificados em ovos sujos, quebrados, trincados e ovos sem casca, em toda a produção durante a pesquisa. Foi feita a determinação do peso do ovo (g) utilizando-se uma balança eletrônica marca Marte modelo 5000, considerando o peso dos ovos um valor de referência na avaliação do melhor resultado econômico.

Durante o experimento, foram verificadas a temperatura ambiente e a umidade relativa do ar com a utilização de termômetros de bulbos seco e úmido para aferição da temperatura e umidade respectivamente. Para análise estatística dos dados, foram obtidas a média, mediana, desvio-padrão, coeficiente de variação, mínimo e máximo (Técnicas de estatística descritiva) e utilizados o F (ANOVA) e no caso de diferença significativa, foi utilizado o teste de comparações múltiplas (pareadas) de Tukey (Técnicas de estatística inferencial). O software utilizado para a obtenção dos cálculos estatísticos foi o SAS (Statistical Analysis System). A margem de erro utilizada na decisão dos testes estatísticos foi de 5,0\%.

\section{Resultados e Discussão}

A média do peso do ovo tipo jumbo variou de $84,58 \mathrm{~g}$ (14 aves/gaiola) a 88,67 g (12 aves/gaiola) sem diferença entre os grupos $(\mathrm{P}>0,05)$. A média do peso do ovo tipo extra variou de 66,38 g (14 aves/gaiola) a 67,47 g (8 aves/gaiola), sendo significante $(\mathrm{P}<0,05)$. A média do peso do ovo tipo 
primeira variou de 59,67 g(14 aves/gaiola) a 60,26 g (08 aves/ gaiola) e não diferiu entre os grupos ( $\mathrm{P}>0,05)$. A média do peso do ovo tipo segunda variou de 50,62 g (10 aves/gaiola) até 52,96 g (12 aves/gaiola) e foi significativa $(\mathrm{P}<0,05)$.

Existem diferenças quanto ao peso dos ovos tipo extra e tipo segunda (Tabela 1) entre as densidades ave/alojada estudadas, com tendência de aumento do peso dos ovos a medida que o número de aves diminuiu. Esses resultados reforçam os achados de Pavan et al. (2005), que estudaram densidade de alojamento entre 562,15 e $375,00 \mathrm{~cm}^{2} /$ ave e observaram diminuição no peso dos ovos à medida que diminuíram o espaço das aves nas gaiolas, porém são contrários aos resultados obtidos por Roush et al. (1984), que estudaram densidade de alojamento de aves variando entre 516 e $310 \mathrm{~cm}^{2} /$ ave e relataram diminuição na produção das aves e aumento no peso dos ovos à medida que a densidade ave/alojada aumentou. A média do peso dos ovos tipos jumbo e primeira indicou ausência de efeito da densidade populacional na gaiola sobre o peso dos ovos, resultados que reforçam os achados de Martim et al. (1976), Cunningham (1982), Goodling et al. (1984), Mench et al. (1986), Lee (1989), Al Rawi et al. (1995), Carey et al. (1995) de que não há efeito da densidade populacional das aves sobre o peso dos ovos. Por outro lado, divergem dos resultados relatados por Davami et al. (1987), que constataram que o peso dos ovos diminui conforme aumenta a densidade das aves na gaiola.

A média de tipos de ovos produzidos por ave variou (Tabela 2) entre: tipo jumbo de 0,73 (14 aves/gaiola) a 1,20 (10 aves/gaiola); tipo extra de 23,29 (8 aves/gaiola) a 25,22 (10 aves/gaiola); tipo primeira de 109,42 (10 aves/gaiola) a 112,42 (8 aves/gaiola) e tipo segunda de 1,14 (08 aves/ gaiola) a 4,00 (14 aves/gaiola). O total de ovos produzidos por ave variou de 137,15 (12 aves/gaiola) a 138,88 (14 aves/ gaiola). A única diferença ocorreu entre o grupo 4 e os grupos 1 e 3 nos ovos tipos segunda $(\mathrm{P}<0,05)$.

A densidade não teve influência sobre a produção diária dos ovos tipo: jumbo, extra e primeira, mas interferiu na produção de ovos tipo segunda. Esses resultados diferem dos relatados por Marks et al. (1970), Dorminey \& Arscott (1971), Wells (1971), Roush et al. (1984), Mench et al. (1986), Lee (1989), Craig \& Milliken (1989) e Carey et al. (1995), que afirmam não haver influência da densidade populacional sobre a produção diária dos ovos, e reforçam os achados de Albuquerque (2004), Barbosa Filho et al. (2007), Campos (2004), Mashaly (2004) e Furlan et al. (2006), que relataram queda na produção diária de ovos à medida que aumentou a densidade de aves nas gaiolas. $\mathrm{O}$ fato de não ter ocorrido

Tabela 1 - Peso dos ovos de acordo com o tipo de ovo e o grupo de aves em diferentes densidades de alojamento

\begin{tabular}{|c|c|c|c|c|c|c|}
\hline \multirow{2}{*}{ Tipo do ovo } & & \multicolumn{4}{|c|}{ Densidade de alojamento ( $\mathrm{cm}^{2} /$ ave) } & \multirow[b]{2}{*}{ Valor de $\mathrm{P}$} \\
\hline & & 625 & 500 & 416,6 & 357,14 & \\
\hline \multirow[t]{7}{*}{ Jumbo } & Média (g) & 84,96 & 88,67 & 86,04 & 84,58 & $\mathrm{p}^{(1)}=0,0629 \mathrm{~ns}$ \\
\hline & Mediana (g) & 82,90 & 88,00 & 85,50 & 85,00 & \\
\hline & Desvio-padrão (g) & 7,57 & 11,27 & 7,59 & 5,69 & \\
\hline & CV $(\%)$ & 8,91 & 12,71 & 8,82 & 6,73 & \\
\hline & Mínimo (g) & 71,90 & 75,80 & 74,40 & 71,20 & \\
\hline & Máximo (g) & 103,00 & 159,40 & 114,05 & 95,90 & \\
\hline & $\mathrm{N}$ & 32 & 60 & 48 & 49 & \\
\hline \multirow[t]{7}{*}{ Extra } & Média & $67,47(\mathrm{~A})$ & $66,98(\mathrm{AB})$ & $67,23(\mathrm{~A})$ & $66,38(B)$ & $\mathrm{p}^{(1)}=0,0007^{*}$ \\
\hline & Mediana & 67,78 & 67,20 & 67,61 & 66,73 & \\
\hline & Desvio-padrão & 2,50 & 3,06 & 1,42 & 1,98 & \\
\hline & CV (\%) & 3,70 & 4,56 & 2,12 & 2,98 & \\
\hline & Mínimo & 43,99 & 42,39 & 62,30 & 55,57 & \\
\hline & Máximo & 70,70 & 79,96 & 72,20 & 73,20 & \\
\hline & $\mathrm{N}$ & 141 & 135 & 143 & 140 & \\
\hline \multirow[t]{7}{*}{ Primeira } & Média & 60,26 & 60,09 & 60,24 & 59,67 & $\mathrm{p}^{(1)}=0,2607 \mathrm{~ns}$ \\
\hline & Mediana & 60,50 & 60,20 & 60,70 & 60,30 & \\
\hline & Desvio-padrão & 1,95 & 2,19 & 1,84 & 4,67 & \\
\hline & CV (\%) & 3,24 & 3,65 & 3,06 & 7,83 & \\
\hline & Mínimo & 46,10 & 49,40 & 52,70 & 7,10 & \\
\hline & Máximo & 63,60 & 74,90 & 71,30 & 70,60 & \\
\hline & $\mathrm{N}$ & 150 & 150 & 150 & 150 & \\
\hline \multirow[t]{7}{*}{ Segunda } & Média & $52,44(\mathrm{~A})$ & $50,62(B)$ & $52,96(\mathrm{~A})$ & $52,85(\mathrm{~A})$ & $\mathrm{p}^{(1)}<0,0001^{*}$ \\
\hline & Mediana & 52,08 & 50,50 & 52,00 & 52,00 & \\
\hline & Desvio-padrão & 2,95 & 1,80 & 5,09 & 2,87 & \\
\hline & CV (\%) & 5,62 & 3,56 & 9,61 & 5,43 & \\
\hline & Mínimo & 47,45 & 45,83 & 46,65 & 47,50 & \\
\hline & Máximo & 59,50 & 55,60 & 76,05 & 63,00 & \\
\hline & N & 48 & 82 & 41 & 117 & \\
\hline
\end{tabular}

* Diferença significativa a 5,0\%; ns = não-significativa; ${ }^{1}$ Pelo teste $\mathrm{F}($ ANOVA). 
diminuição na produção de ovos dos principais tipos de ovos (jumbo, extra e primeira) está relacionado à temperatura, pois, mesmo com o aumento na densidade de alojamento, as aves estavam em lugar bastante arejado e não houve aumento da temperatura ambiente que provocasse estresse nas aves ou interferisse na produção dos principais tipos de ovos.

A média de ovos por semana variou de 6,48 a 6,52 ovos (Tabela 3) e não diferiu entre os grupos ( $\mathrm{P}>0,05)$.

O total de ovos produzidos variou de 137,15 a 138,87 ovos e o custo da ração foi igual entre os grupos (Tabela 4). A razão custo total da ração/total de ovos foi igual a $\mathrm{R} \$ 0,05$ por ovo em cada grupo, enquanto a razão total de ovos por custo e ave variou de 19,51 a 19,75 ovos para R \$1,00 utilizado.

O consumo de ração por ave variou entre 15,27 e $15,29 \mathrm{~kg}$ (Tabela 5) e não diferiu entre os grupos. A massa de ovos produzidos variou de $8,43 \mathrm{~kg}$ a $8,50 \mathrm{~kg}$, resultando numa conversão alimentar que variou de 1,80 a 1,81 (Tabela 5), sem diferença entre os grupos.

A massa de ovos produzida não foi alterada pelas densidades ave/alojada estudadas e, embora o peso dos ovos tenha sido maior na densidade de $500 \mathrm{~cm}^{2} /$ ave (10 aves/ gaiola), este aumento não foi siginificativo. Entretanto houve tendência de elevação da massa de ovos produzidos com a diminuição da densidade, divergindo dos relatos de Carey et al. (1995), que observaram tendência de diminuição da massa de ovos produzidos à medida que diminuíram a densidade das aves na gaiola e confirmam também os achados de Okpokho et al. (1987), que, utilizando densidades de 348, 464 e $580 \mathrm{~cm}^{2} /$ ave com aves entre 22 e 70 semanas, também descreveram redução na massa de ovos produzida com o aumento da densidade de alojamento. A conversão alimentar por quilograma de ração e o consumo de ração não sofreram alterações nas diferentes densidades estudadas. Esses resultados estão de acordo com os de Roush et al. (1984); Mench et al. (1986), Lee(1989) e Carey etal.(1995),que também relataram não haver influência da densidade de alojamento sobre a conversão alimentar e o comportamento das aves.

Os resultados da conversão alimentar foram diferentes dos observados por Hill (1977); Albuquerque (2004), Campos (2004), Conto (2004), Mashaly (2004) e Furlan et al. (2006) que descreveram haver melhoria da conversão alimentar por quilo de ração consumida com o aumento da densidade ave/alojada. Cunningham \& Ostrander (1982); Davami et al. (1987) e Cunningham et al. (1988), no entanto, constataram melhoria da conversão alimentar por dúzia e por quilograma de ovos produzidos quando diminuíram a densidade de alojamento das aves.

As médias de ovos trincados e sujos foram mais elevadas no grupo com 8 aves/gaiola ( $625 \mathrm{~cm}^{2} /$ ave), também neste grupo de aves a média de ovos impróprios para consumo foi mais baixa. A média de ovos com outra condição foi mais elevada no grupo de 12 aves/gaiola (416,6 $\mathrm{cm}^{2} /$ ave). A soma dos ovos impróprios para consumo foi maior no grupo de 8 aves/gaiola ( $625 \mathrm{~cm}^{2} /$ ave) e menor no grupo de 12 aves/gaiola (416,6 $\mathrm{cm}^{2} /$ ave). Não houve diferença entre os grupos para nenhuma destas variáveis.

Tabela 2 - Produção média de ovos por ave alojada segundo o tipo de ovo e o grupo de aves em diferentes densidades de alojamento

\begin{tabular}{|c|c|c|c|c|c|c|c|c|c|}
\hline \multirow{3}{*}{ Tipo do ovo } & \multicolumn{8}{|c|}{ Densidade de alojamento ( $\mathrm{cm}^{2} / \mathrm{ave}$ ) } & \multirow{3}{*}{ Valor de $\mathrm{P}$} \\
\hline & \multicolumn{2}{|c|}{625} & \multicolumn{2}{|c|}{500} & \multicolumn{2}{|c|}{416,6} & \multicolumn{2}{|c|}{357,14} & \\
\hline & $\mathrm{n}$ & Média & $\mathrm{n}$ & Média & $\mathrm{n}$ & Média & $\mathrm{n}$ & Média & \\
\hline Extra & 141 & 23,29 & 135 & 25,22 & 143 & 24,97 & 140 & 24,29 & $\mathrm{p}^{(1)}=0,9692 \mathrm{~ns}$ \\
\hline Primeira & 150 & 112,42 & 150 & 109,42 & 150 & 110,17 & 150 & 109,86 & $\mathrm{p}^{(1)}=0,9603 \mathrm{~ns}$ \\
\hline Segunda & 48 & $1,58(\mathrm{~A})$ & 82 & $2,80(\mathrm{AB})$ & 41 & $1,14(\mathrm{~A})$ & 117 & $4,00(B)$ & $\mathrm{p}^{(1)}=0,0085^{*}$ \\
\hline Total de ovos & 150 & 138,04 & 150 & 138,64 & 150 & 137,15 & 150 & 138,88 & $\mathrm{p}^{(1)}=0,2038 \mathrm{~ns}$ \\
\hline
\end{tabular}

* Diferença significativa a 5,0\%; ns = não-significativa; ${ }^{1}$ Pelo teste $\mathrm{F}$ (ANOVA).

Tabela 3 - Número de ovos produzidos por semana, segundo o grupo de aves em diferentes densidades de alojamento

\begin{tabular}{lcccc}
\hline & \multicolumn{4}{c}{ Densidade de alojamento $\left(\mathrm{cm}^{2} / \mathrm{ave}\right)$} \\
\cline { 2 - 4 } & 625 & 500 & 416,6 & 357,14 \\
\hline Média & 6,50 & 6,48 & 6,47 & 6,52 \\
Mediana & 6,58 & 6,52 & 6,56 & 6,56 \\
Desvio-padrão & 0,34 & 0,29 & 0,23 & 0,19 \\
Coeficiente de variação & 5,23 & 4,46 & 3,53 & 2,86 \\
Mínimo & 5,67 & 5,45 & 5,90 & 6,10 \\
Máximo & 6,92 & 6,77 & 6,75 & 6,83 \\
N & 21 & 21 & 21 & 21 \\
\hline
\end{tabular}

* Diferença significativa a 5,0\%; ns = não-significativa; ${ }^{1}$ Pelo teste $\mathrm{F}$ (ANOVA). 
A porcentagem de ovos imprestáveis para o consumo humano (Tabela 6) não foi influenciada pela densidade de alojamento de aves nesta pesquisa. Na literatura, alguns autores afirmam que os parâmetros de qualidade dos ovos, incluindo porcentagem de ovos quebrados, geralmente não são afetados pela densidade na gaiola (Robinson, 1979; Pavan et al., 2005). No entanto, Cunningham (1982), Davami et al. (1987), Cunningham et al. (1988), Brake \& Peebles (1992), Garcia et al. (1993), Mench et al. (1986), Albuquerque (2004), Mashaly (2004) e Furlan et al. (2006) relataram que menor resistência da casca foi encontrada no grupo de maior densidade de alojamento. Esses dados reforçam o entendimento de que, quando a ave tem espaço maior na gaiola, poderia haver maior contato da ave com o ovo, proporcionando assim maior número de ovos trincados, quebrados e sujos.

A produção de ovos limpos é importante, visto que os classificados como qualidade inferior, na maioria das vezes não chega ao consumidor final. Para melhor produtividade e aumento na qualidade do produto, são esperados sistemas de produção que não agridam o ambiente e assegurem o bem-estar das aves. Para melhor avaliar as atuais estratégias de produção de aves, é necessário ampliar o conhecimento sobre seus comportamentos e bem-estar, independentemente dos sistemas de criação. Só assim pode-se interferir de forma adequada, propondo novos sistemas de produção que proporcionem instalações e manejos adequados.

Tabela 4 - Média de ovos produzidos, custo total da ração, relação custo total da ração/total de ovos produzidos, relação total de ovos produzidos/custo total da ração por animal segundo o grupo de aves em diferentes densidades de alojamento

\begin{tabular}{|c|c|c|c|c|}
\hline & \multicolumn{4}{|c|}{ Densidade de alojamento ( $\mathrm{cm}^{2} /$ ave) } \\
\hline & 625 & 500 & 416,6 & 357,14 \\
\hline Total de ovos produzidos & 138,04 & 138,64 & 137,15 & 138,87 \\
\hline Custo total da ração (R\$) & 7,03 & 7,03 & 7,03 & 7,03 \\
\hline Razão: custo total da ração/total de ovos produzidos & 0,05 & 0,05 & 0,05 & 0,05 \\
\hline Razão: Total de ovos produzidos/custo total da ração & 19,63 & 19,72 & 19,51 & 19,75 \\
\hline
\end{tabular}

Tabela 5 - Avaliação da conversão alimentar segundo o grupo de aves em diferentes densidades de alojamento

\begin{tabular}{lcccc}
\hline & \multicolumn{4}{c}{ Densidade de alojamento (cm²/ave) } \\
\cline { 2 - 5 } Item & 625 & 500 & 416,6 & 357,14 \\
\hline Ração consumida/ave & 15,29 & 15,29 & 15,29 & 15,27 \\
Massa de ovos/ave & 8,48 & 8,50 & 8,44 & $\mathrm{p}^{(1)}=0,9965 \mathrm{~ns}$ \\
Conversão alimentar & 1,80 & 1,80 & 1,81 & $\mathrm{p}^{(1)}=0,9999 \mathrm{~ns}$ \\
\hline
\end{tabular}

${ }^{1}$ Pelo teste F (ANOVA); ns = não-significativa.

Tabela 6 - Média de ovos não-conformes por ave em diferentes densidades de alojamento

\begin{tabular}{|c|c|c|c|c|c|}
\hline \multirow[b]{2}{*}{ Item } & \multicolumn{5}{|c|}{ Densidade de alojamento ( $\mathrm{cm}^{2} / \mathrm{ave}$ ) } \\
\hline & 625 & 500 & 416,6 & 357,14 & Valor de $\mathrm{P}$ \\
\hline Trincados & 1,17 & 0,60 & 0,71 & 0,78 & $\mathrm{p}^{(1)}=0,0593 \mathrm{~ns}$ \\
\hline Sujos & 0,98 & 0,83 & 0,64 & 0,94 & $\mathrm{p}^{(1)}=0,4014 \mathrm{~ns}$ \\
\hline Outros & 0,19 & 0,12 & 0,42 & 0,03 & $\mathrm{p}^{(1)}=0,0541 \mathrm{~ns}$ \\
\hline Soma & 2,48 & 1,72 & 1,60 & 1,98 & $\mathrm{p}^{(1)}=0,0767 \mathrm{~ns}$ \\
\hline
\end{tabular}

${ }^{1}$ Pelo teste $\mathrm{F}$ (ANOVA); ns = não significativa.

\section{Conclusões}

Nas condições em que a pesquisa foi realizada, a utilização de gaiolas com diferentes densidades de alojamento na fase de produção não prejudica os parâmetros zootécnico e econômico da produção.

\section{Literatura Citada}

ALBUQUERQUE, R. Tópicos importantes na produção de poedeiras comerciais. Avicultura Industrial, v.1121, n.95, 2004.

ADAMS, A.W.; CRAIG, J.V. Effect of crowding and cage shape on productivity and profitability of caged layers: a survey. Poultry Science, v.64, n.2, p.238-242, 1985. 
AL-RAWI, B.; CRAIG, J.V.; ADAMS, A.W. Agonistic behavior and egg production of caged layers: genetic strain and group size effects. Poultry Science, v.55, n.2, p.796-807, 1976.

BARBOSA FILHO, J.A.D.; SILVA, I.J.O.; SILVA, M.A.N. et al. Avaliação dos comportamentos de aves poedeiras utilizando seqüência de imagens. Engenharia Agrícola, v.27, n.1, p.93-99, 2007.

BRAKE, J.D.; PEEBLES, E.D. Laying hen performance as affected by diet and caging density. Poultry Science, v.71, n.6, p.945-950, 1992.

CAMPOS, S.S. Fatores responsáveis pelo progresso nas instalações avícolas. Avicultura Industrial, v.1121, n.95, 2004.

CAREY, J.B. Effects of pullet-stocking density on performance of laying hens. Poultry Science, v.66, n.8, p.1283-1287, 1992.

CAREY, J.B.; KUO, F.L.; ANDERSON, K.E. Effects of cage population on the productive performance of layers. Poultry Science, v.74, n.4, p.633-637, 1995.

CONTO, LA. Avicultura de postura. Avicultura Industrial, v.1121, n.95, 2004.

CRAIG, J.V.; MILLIKEN, G.A. Further studies of density and group size effects in caged hens of stocks differing in fearful behavior: productivity and behavior. Poultry Science, v.68, n.1. p.9-16, 1989 .

CUNNINGHAM, D.L. Cage type and density effects on performance and economic factors of caged layers. Poultry Science, v.61, n.10, p.1944-1949, 1982.

CUNNINGHAM, D.L.; OSTRANDER, C.E. The effects of strain and cage shape and density on performance and fearfulness of white leghorn layers. Poultry Science, v.61, n.2, p.239-243, 1982.

CUNNINGHAM, D.L.; VAN TIENHOVEN, A.; GVARYAHU, G. Population size, cage area, and dominance rank effects on productivity and web-being of laying hens. Poultry Science, v.67, n.3, p.399-406, 1988.

DAVAMI, A.; WINELAND, M.J.; JONES, W.T. et al. Effects of population size, floor space, and feeder space upon productive performance, external appearance, and plasma corticosterone concentration of laying hens. Poultry Science, v.66, n.2, p.251-257, 1987.

DORMINEY, R.W.; ARSCOTT, G.H. Effects of bird density, nutrient density and perches on the performance of caged white leghorn layers. Poultry Science, v.50, n.2, p.619- 626, 1971.

FURLAN, R.L.; MACARI, M.; MATEUS, J.R. [2006]. Bem estar das aves e suas implicações sobre o desenvolvimento e produção. Disponível em: <http://www.engomix.com/bem_ estar_das_aves_P_artigos_17_AVG.htm>. Acesso em: 21/10/2006.
GARCIA, E.A.; AGUIAR, I.S.; POLITI, E.S. et al. Efeito da taxa de lotação da gaiola sobre a produtividade de poedeiras brancas. In: CONFERÊNCIA APINCO DE CIÊNCIA E TECNOLOGIA AVÍCOLAS, 93., 1993, Santos. Anais... Santos: 1993. p.71.

GOODLING, A.C.; SATTERLEE, D.G.; CERNIGLIA, G.J. et al. Influence of toe-clipping and stocking density on laying hen performance. Poultry Science, v.63, n.9, p.1722-1731, 1984.

HILL, A.T. The effects of space allowance and group size on egg production traits and profitability. British Poultry Science, v.17, p.483-492, 1977.

LEE, K. Laying performance and fear response of white leghorns as influenced by floor space allowance and group size. Poultry Science, v.68, n.10, p.1333-36, 1989.

MARKS, H.L.; TINDELL, L.D.; OLWE, R.H. Performance of egg production stocks under three cages densities. Poultry Science, v.49, n.4, p.1094-1100, 1970.

MARTIN, G.A.; WEST, J.R.; MORGAN, G.W. Cage shape and crowding effects on layers. Poultry Science, v.55, n.5, p.2061, 1976.

MASHALY, M.M.; HENDRICKS III, G.L.; KALAMA, M.A. et al. Effect of heat stress on production parameteres and immune responses of commercial laying hens. Poultry Science, v.83, p.889-894, 2004.

MENCH, J.A.; TIENHOVEN, A.V.; MARSH, J.A. et al. Effects of cage and floor pen management on behavior, production, and physiological stress responses of laying hens. Poultry Science, v.65, n.6, p.1058-1069, 1986.

OKPOKHO, N.A.; CRAIG, J.V.; MILLIKEN, G.A. Density and group size effects on cage hens of two genetic stocks differing in escape and avoidance behavior. Poultry Science, v.66, n.12, p.1905-1910, 1987.

PAVAN, A.C.; GARCIA, E.A.; MÓRI, C. et al. Efeito da densidade na gaiola sobre o desempenho de poedeiras comerciais nas fases de cria, recria e produção. Revista Brasileira de Zootecnia, v.34, n.4, p.1320-1328, 2005.

ROBINSON, D. Effects of cage shape, colony size, floor area and cannibalism preventatives on laying performance. British Poultry Science, v.20, p.345-356, 1979

ROUSH, W.B.; MASHALY, M.M.; GRAVES, H.B. Effect of increased bird population in a fixed cage area on production and economic responses of single comb white leghorn laying hens. Poultry Science, v.63, n.1, p.45-48, 1984.

WELLS, R.G. Studies on stocking arrangements for caged layers. World's Poultry Science Journal, v.27, p.361-366, 1971. 